\title{
Gestión sostenible para la producción de biofungicidas y fortalecimiento del sector de bioinsumos agrícolas venezolano
}

\section{(Sustainable management for the production of biofungicides and strengthening of the Venezuelan agricultural bio-inputs sector)}

\author{
Griselda Tamara Colmenares Lima, ${ }^{1}$ Miguel Asdrúbal Arcia Montezuma ${ }^{1}$
}

\begin{abstract}
Resumen
El propósito de este trabajo fue diseñar una propuesta de gestión para la producción de biofungicidas, fundamentada en normas internacionales relativas a la calidad, ambiente y seguridad, y salud ocupacional, y orientada a fortalecer al sector de bioinsumos agrícolas venezolano, en la elaboración de productos de excelente calidad obtenidos bajo esquemas de producción sostenibles. Se realizó un diagnóstico para establecer las conformidades de la gestión actual de los laboratorios dedicados a esta actividad con lo establecido en las normas, además de un diagnóstico situacional para la adopción de la propuesta. Es una investigación aplicada, de campo y nivel de estudio descriptivo. Los datos se recolectaron entre abril y septiembre de 2018. Las técnicas empleadas fueron la encuesta y observación estructurada; y como instrumentos el cuestionario y la lista de verificación. Se generó un modelo de gestión sostenible que promueve la adecuación del quehacer y ser de la organización, con el deber ser, constituyéndose en un insumo para sentar las bases que propicien el reconocimiento nacional e internacional de los laboratorios y de la plataforma para asegurar el cumplimiento de los requisitos del proceso de registro, principalmente en lo relacionado con la garantía de la calidad, condiciones de seguridad del proceso de producción, documentación requerida y evaluación del producto.
\end{abstract}

\section{Palabras clave}

gestión; biofungicidas; sostenibilidad.

\begin{abstract}
The purpose of this work was the design of a management proposal for the production of biofungicides, based on international standards related to quality, environment and safety, and occupational health, aiming at strengthening the Venezuelan agricultural bio-inputs sector, in the production of excellent quality products obtained under sustainable production schemes. A diagnosis was made to establish the conformities of the current management of the laboratories dedicated to this activity with the provisions in the standards, besides of a situational diagnosis for the adoption of the proposal. It is an applied investigation, of field and level of descriptive study; the data were collected between April-September 2018 using the survey and structured observation as techniques, and the questionnaire and checklists as instruments. A sustainable management model was generated to promote the adequacy of the organization's task and being, with the duty to be, constituting an input to lay the foundations that propitiate the national and international recognition of the laboratories and platform, to ensure compliance with the requirements of the registration process, mainly in relation to quality assurance, safety conditions of the production process, required documentation and evaluation of the product.
\end{abstract}

\section{Keywords}

management; biofungicides; sustainability.

\section{Introducción}

La incidencia de plagas y enfermedades en los cultivos y el uso indebido de agentes químicos para su control, son factores que limitan el desarrollo sostenible de la actividad agrícola, además de incidir negativamente sobre la seguridad alimentaria y la preservación del ambiente. 
Desde la década de 1960, la gestión de plagas en los países industrializados se ha basado en el uso de agroquímicos, mejoramiento genético de las variedades, la mecanización, el riego y la nutrición de los cultivos, lo cual ha contribuido a aumentar los rendimientos en casi un $70 \%$ en Europa y un 100 \% en Estados Unidos (Chandler et al., 2011).

Sin embargo, el uso de los agentes químicos ha sido intensivo e indiscriminado, lo que ha llevado al replanteamiento de esta práctica y a la necesidad de buscar opciones ambientalmente más amigables. Está demostrado que, debido a la toxicidad elevada de algunos agentes y su persistencia en el medio, se generan problemas de salud en los humanos y animales, eliminación de fauna benéfica, desarrollo de resistencia por parte de las plagas y resurgencia de organismos nocivos, además de contribuir con la reducción de la biodiversidad natural y la contaminación de los alimentos y el ambiente (Chandler et al., 2011).

En ese sentido, se da especial énfasis en señalar al control biológico como una alternativa viable, eficiente y opcional al control químico de plagas y enfermedades en los cultivos, enmarcada en los principios de una agricultura sustentable y, aunque ya en 1935 se conocía el potencial de los hongos para controlar insectos, no fue sino hasta 1980 que esta práctica adquiere mayor relevancia, por la preocupación de la preservación del ambiente y la inocuidad alimentaria (Arcia y Bautista, 2009; Falconí, 2014; Jaronski, 2013; Paleologos y Flores, 2014; Reyes, Rincón, López, Evangelista y Quiñones, 2015; Serrano y Galindo, 2007).

El control biológico en su sentido más amplio, es definido como el uso de organismos vivos o de sus metabolitos, para el control de plagas. Es una estrategia válida para restaurar la biodiversidad en los ecosistemas agrícolas, ya que promueve el uso racional y adecuado de organismos o microorganismos benéficos denominados "biocontroladores o agentes de control biológico", entre ellos los biofungicidas, seleccionados por su alta eficiencia e inocuidad para disminuir o regular la densidad poblacional de un organismo o microorganismo plaga o perjudicial, en un proceso de equilibrio poblacional y ecológico (Arcia y Bautista, 2009; Cock, 1996; FAO, 2006; INSAI, 2016; Paleólogos y Flores, 2014; Reyes et al., 2015).

No obstante, las bondades del uso de estos agentes para el control de plagas agrícolas, su producción en América Latina y el Caribe, desde el punto de vista agronómico, requiere enfrentar desafíos, limitantes y debilidades, tales como: desarrollar productos de calidad que estén disponibles en el mercado, metodologías para su producción a gran escala y transferencia al sector privado, formulaciones que promuevan la facilidad de uso y su conservación, así como protocolos para la evaluación de la calidad. Asimismo, se requieren métodos para la integración de los agentes de control biológico en los sistemas productivos y elaboración de normativas para el registro y comercialización de los mismos (Bettiol, Rivera, Mondino, Montealegre y Colmenárez, 2014).

En ese orden de ideas se encuentran países como Argentina, Brasil, Chile, Colombia, Ecuador, Honduras, Costa Rica, México, Cuba, Uruguay, entre otros, donde con mayor o menor avance tecnológico se producen agentes de control biológico, coincidiendo en casi todos los casos con lo referido por Bettiol et al. (2014), respecto a la necesidad de afrontar el reto que conlleva producir biocontroladores a escala industrial con criterio de sostenibilidad.

En Venezuela, el control biológico ha seguido el mismo camino que la mayoría de los países latinoamericanos y es hoy cuando se presenta como columna vertebral en la mayoría de los programas de manejo integrado de plagas con las nuevas políticas de recuperación ambiental y desarrollo sustentable de la agricultura; siendo a partir de 2013 cuando se incrementó el uso de insumos biológicos como alternativas ya comprobadas gracias a los aportes científicos y técnicos de investigadores y líderes en el área. (Zambrano, Goyo, Jiménez y Zambrano, 2014). 
Sin embargo, en el país no existe una legislación específica que regule la producción de los agentes biológicos ni se dispone de protocolos y parámetros oficiales estandarizados en los cuales apoyarse para llevar a cabo los planes de control de la producción, calidad, comercialización, almacenamiento, conservación y aplicación en campo; existiendo mucha heterogeneidad en los criterios y técnicas usadas para la obtención y uso exitoso de estos productos, lo cual, además de ser una limitante, promueve la distribución a nivel nacional de productos que carecen de la certificación científica necesaria y del registro correspondiente (Zambrano et al., 2014).

Adicionalmente, no se cuenta con procedimientos de control y seguimiento documentados y sistematizados que permitan establecer el nivel de acompañamiento al agricultor y de conformidad de uso por parte de estos y por tanto, de los resultados obtenidos.

Como un aporte a la solución de la problemática planteada, se propuso desarrollar esta investigación con la finalidad de brindar una herramienta de gestión enmarcada en la Legislación Nacional y fundamentada en las Normas Internacionales ISO 9001:2015, ISO 14001:2015 y OSHAS 18001:2007, relativas a la Calidad, al Ambiente y a la Seguridad y Salud Ocupacional (SSO). (ISO, 2015a; ISO, 2015b y OSHAS, 2008).

El objetivo fundamental que se planteó en este trabajo fue determinar las conformidades y no conformidades de la gestión actual para la producción de biofungicidas en los laboratorios públicos y privados dedicados a esta actividad en Venezuela, de acuerdo con lo establecido en las Normas Internacionales ISO 9001:2015, ISO 14001:2015 y OSHAS 18001:2007, del análisis de los resultados del diagnóstico, se establecieron las fortalezas, debilidades, oportunidades y amenazas para la adopción de una propuesta de gestión fundamentada en dichas normas.

Con esta propuesta se espera contribuir con el desarrollo y establecimiento de normas nacionales para el registro y comercialización de los biofungicidas, así como con el mejoramiento continuo de la gestión de los laboratorios y empresas del sector para que alcancen en el tiempo un desempeño orientado a la sostenibilidad de sus procesos. Esto les permitirá asegurar la calidad del servicio que prestan para el sector productivo nacional, además de que podrá sentar las bases que propicien el reconocimiento nacional e internacional sobre su competencia técnica, imparcialidad e integridad de los resultados; permitiéndoles así acceder y competir en mercados cada vez más exigentes, mejorando su eficiencia operacional y garantizando la satisfacción del cliente, debido a la autoexigencia de suministrar productos y servicios de calidad que resguarden la seguridad de las personas y contribuyan a garantizar la inocuidad de los alimentos y la preservación del ambiente.

\section{Metodología}

\subsection{Tipo y nivel de la investigación}

De acuerdo con Balestrini (2001) y Hernández, Fernández y Baptista (2014), el presente trabajo corresponde a una investigación aplicada, de campo, se realizó apoyado en una investigación documental, donde se definieron un conjunto de variables sin manipulación intencional o deliberada, con la finalidad de describirlas con precisión, por medio de la observación de situaciones ya existentes y se recolectaron los datos directamente de la realidad objeto de estudio sin posibilidades de generalización a poblaciones enteras.

Por su dimensión temporal es una investigación transversal descriptiva, ya que los datos se recolectaron en un único momento, esto es en el período de tiempo entre abril-septiembre de 2018, y las variables se describen sin considerar cómo evolucionan, tal como lo plantea Her- 
nández et al. (2014), quien refiere que "los diseños de investigación transeccional o transversal recolectan datos en un solo momento, en un tiempo único. Su propósito es describir variables y analizar su incidencia e interrelación en un momento dado" (p. 208).

En lo que se refiere al alcance de la investigación, se puede considerar que es un estudio de tipo descriptivo, definido por Hernández et al. (2014), como estudios que miden, evalúan o recolectan datos sobre diversas variables, aspectos, dimensiones o componentes del fenómeno a investigar.

\subsection{Población y muestra}

La unidad de análisis del presente trabajo de investigación fue la gestión para la producción de bioplaguicidas microbianos a partir de hongos entomopatógenos y antagonistas para el control de plagas agrícolas en los cultivos, por lo que la población estuvo constituida por los catorce laboratorios públicos y privados del país que actualmente están dedicados a esta actividad.

La muestra considerada fue del tipo no probabilística, clasificada como Muestra Dirigida de Expertos, según Hernández et al. (2014), ampliamente utilizada cuando se pretende mejorar un proceso industrial o de calidad, en virtud de que la información fue suministrada por el personal de los laboratorios especialista en el área. La muestra estuvo conformada por nueve de los catorce expertos dedicados a la producción de biofungicidas, es decir, el 64,3\% de la población.

\subsection{Operacionalización de Variables}

Para el diseño del cuestionario utilizado en la recolección de los datos se realizó previamente la operacionalización de variables de acuerdo con lo mostrado en la Tabla 1, donde se pueden observar las dimensiones, subdimensiones, indicadores, fuente y técnicas e instrumentos que se emplearon. Las dimensiones establecidas para el análisis de la gestión son: Calidad, Ambiente y SSO, de acuerdo con las normas ISO 9001:2015, 14001:2015 Y OSHAS 18001:2007, tomadas como referencia. Las subdimensiones e indicadores se establecieron de acuerdo con los requerimientos de dichas normas.

Tabla 1. Operacionalización de variables para el análisis de la gestión

\begin{tabular}{|c|c|c|c|c|c|c|}
\hline $\begin{array}{l}\text { OBJETIVO } \\
\text { ESPECÍFICO }\end{array}$ & VARIABLES & DIMENSIONES & SUB DIMENSIONES & $\begin{array}{l}\text { DEFINICIÓN } \\
\text { OPERACIONAL } \\
\text { INDICADORES }\end{array}$ & FUENTE & $\begin{array}{c}\text { TÉCNICAS E } \\
\text { INSTRUMENTOS }\end{array}$ \\
\hline 1 & $\begin{array}{l}\text { GESTIÓN PARA LA } \\
\text { PRODUCCIÓN DE } \\
\text { BIOFUNGICIDAS }\end{array}$ & $\begin{array}{l}\text { CALIDAD } \\
\text { AMBIENTE } \\
\text { SEGURIDAD Y SALUD } \\
\text { OCUPACIONAL }\end{array}$ & $\begin{array}{l}\text { CONTEXTO ORGANIZACIÓN } \\
\text { LIDERAZGO } \\
\text { PLANIFICACIÓN } \\
\text { APOYO } \\
\text { OPERACIÓN } \\
\text { EVALUACIÓN } \\
\text { MEJORA } \\
\text { CONTEXTO ORGANIZACIÓN } \\
\text { LIDERAZGO } \\
\text { PLANIFICACIÓN } \\
\text { APOYO } \\
\text { OPERACIÓN } \\
\text { EVALUACIÓN } \\
\text { MEJORA } \\
\text { CONTEXTO ORGANIZACIÓN } \\
\text { LIDERAZGO } \\
\text { PLANIFICACIÓN } \\
\text { APOYO } \\
\text { OPERACIÓN } \\
\text { EVALUACIÓN } \\
\text { MEJORA }\end{array}$ & $\begin{array}{l}\text { REQUISITOS DE } \\
\text { LAS NORMAS ISO } \\
\text { 9001:2015 } \\
\text { BPF } \\
\text { BPL } \\
\text { REQUISITOS DE } \\
\text { LAS NORMAS ISO } \\
\text { 14001:2015 } \\
\\
\text { REQUISITOS DE LAS } \\
\text { NORMAS OSHAS } \\
\text { 18001:2007 }\end{array}$ & $\begin{array}{l}\text { EXPERTOS DE LOS } \\
\text { LABORATORIOS } \\
\text { Y EMPRESAS } \\
\text { PRODUCTORAS DE } \\
\text { BIOFUNGICIDAS } \\
\text { EXPERTOS DE LOS } \\
\text { LABORATORIOS } \\
\text { YEMPRESAS } \\
\text { PRODUCTORAS DE } \\
\text { BIOFUNGICIDAS } \\
\text { EXPERTOS DE LOS } \\
\text { LABORATORIOS } \\
\text { YEMPRESAS } \\
\text { PRODUCTORAS DE } \\
\text { BIOFUNGICIDAS }\end{array}$ & $\begin{array}{l}\text { ENCUESTA/ } \\
\text { CUESTIONARIO } \\
\text { OBSERVACIÓN ES- } \\
\text { TRUCTURADA/LISTA } \\
\text { DE VERIFICACIÓN }\end{array}$ \\
\hline
\end{tabular}




\subsection{Técnica e instrumento para la recolección de los datos}

En esta investigación se utilizó la técnica de la encuesta y la observación estructurada. La encuesta definida por Arias (2006), como "aquella que pretende obtener información que suministra un grupo o muestra de sujetos acerca de sí mismos, o en relación con un tema en particular" (p. 72); y la observación estructurada directa, definida por el mismo autor como aquella que se realiza con unos objetivos preestablecidos y utiliza una guía previamente diseñada en la que se especifican los elementos que se observarán.

La encuesta puede ser oral o escrita. La escrita es la que se realiza mediante un cuestionario y es definido por el mismo autor, como "la modalidad de la encuesta que se realiza en forma escrita mediante un instrumento o formato en papel contentivo de una serie de preguntas. Se le denomina autoadministrado porque puede ser llenado por el encuestado, sin la participación del encuestador" (p. 74).

El instrumento que se empleó para la técnica de la encuesta fue el cuestionario, el cual, según Hernández et al. (2014), es el más utilizado para la recolección de los datos y consiste en un conjunto de preguntas respecto de una o más variables a medir; y para la observación estructurada directa, se utilizó una lista de chequeo o verificación, también conocida como lista de cotejo y en la cual se indica la presencia o ausencia de un aspecto o conducta a ser observada.

En el caso del cuestionario, se elaboraron 150 preguntas a partir de los indicadores derivados de la operacionalización de variables. Para ello se tomaron en cuenta los requisitos de las normas ISO 9001:2015, ISO 14001:2015 y OSHAS 18001:2007. Una vez determinados los indicadores se procedió a la generación de los ítems y establecimiento de la escala de las respuestas, para lo cual se consideró el nivel de medición de las variables. Eso conllevó a presentar a los encuestados una serie de afirmaciones relativas a ciertas actividades, conductas o actitudes, cuya presencia o ausencia permitieron inferir acerca del nivel de conformidad de la subdimensión evaluada con lo establecido en la norma respectiva.

En ese sentido, las preguntas fueron cerradas con categorías u opciones de respuestas previamente delimitadas (Hernández et al., 2014) y de selección simple, ya que se ofrecieron varias opciones para responder solo una (Arias, 2006). El nivel de medición de las variables es ordinal, el cual es definido por Hernández et al. (2014) como aquel en el que "hay varias categorías, pero además estas mantienen un orden de mayor a menor" (p. 215).

Se escogió una escala tipo Likert con cinco opciones de respuestas del 1 al 5. Dependiendo del enunciado o afirmación hecha en cada ítem, el encuestado respondió con alguna de las siguientes categorías: 1. Totalmente en desacuerdo; 2. En desacuerdo. 3. Ni de acuerdo/Ni en desacuerdo. 4. De acuerdo. 5. Totalmente de acuerdo; tal como se muestra en la Tabla 2:

Tabla 2. Categorías de respuestas del instrumento de recolección de datos

\begin{tabular}{|l|l|}
\hline \multicolumn{1}{|c|}{ OPCIONES } & \multicolumn{1}{c|}{ CATEGORÍAS DE RESPUESTAS } \\
\hline 1 & Totalmente en desacuerdo \\
2 & En desacuerdo \\
3 & Ni de acuerdo / Ni en desacuerdo \\
4 & De acuerdo \\
5 & Totalmente de acuerdo \\
\hline
\end{tabular}


Para la interpretación uniforme de los resultados, la concentración de respuestas en la opción 1 significó que el encuestado estaba en total desacuerdo con el enunciado emitido en el cuestionario, en la opción 2 no estar de acuerdo, en la opción 3 no estar de acuerdo o desacuerdo; en la opción 4 estar de acuerdo con el enunciado emitido en el cuestionario y en la opción 5 estar en total acuerdo con las afirmaciones presentadas.

\subsection{Validez y confiabilidad del instrumento de recolección de datos}

La validez se refiere al grado en que un instrumento realmente mide la variable que pretende medir (Hernández et al., 2014). El mismo autor señala que se pueden tener diferentes tipos de evidencia: de constructo, de contenido y de criterio.

En esta investigación se consideró que el instrumento diseñado cuenta con la debida validez en virtud de que se fundamentó estrictamente en cada uno de los requerimientos de las normas ISO consideradas, las cuales son normas internacionalmente aceptadas y validadas.

La confiabilidad de un instrumento de recolección de datos es definida por Hernández et al. (2014), como el grado en el que un instrumento produce resultados consistentes y coherentes. Para su determinación se realizó una prueba piloto y se aplicó el método conocido como Medidas de consistencia interna, Alfa de Cronbach, empleando para la valoración del coeficiente resultante la escala que se muestra en la Tabla 3 donde se señalan los niveles de confiabilidad para cada rango de valores.

Tabla 3. Escala de valoración del coeficiente Alfa de Cronbach

\begin{tabular}{|l|l|}
\hline \multicolumn{1}{|c|}{ RANGOS } & \multicolumn{1}{c|}{ CONFIABILIDAD } \\
\hline 0,81 A 1,00 & Muy alta \\
0,61 a 0,80 & Alta \\
0,41 a 0,60 & Moderada \\
0,21 a 0,40 & Baja \\
0,01 a 0,20 & Muy baja \\
\hline
\end{tabular}

Fuente: Ruiz (s. f.)

El análisis de confiabilidad del instrumento diseñado en la recolección de los datos dio como resultado un valor de alfa de Cronbach de 0,981. Esto indica que la confiabilidad del cuestionario es Muy alta, es decir que los resultados obtenidos son consistentes y coherentes.

Los cuestionarios se aplicaron entre los meses de abril y septiembre de 2018. Se realizó en forma personal y telefónica, con un proceso previo de inducción a los participantes. En algunos casos se hizo por solicitud de la parte interesada en forma presencial y en otros, el cuestionario fue entregado en forma digital y devuelto, posteriormente, una vez resuelto.

\subsection{Técnica para la organización, análisis e interpretación de los resultados}

Para el análisis de los datos se utilizó la estadística descriptiva y se procesaron con el programa InfoStat v. 1/P de la Universidad Nacional de Córdoba, República de Argentina (Infostat, 2002).

Una vez recogidos los cuestionarios se procedió a vaciar la información en una hoja de cálculo para obtener una matriz, la cual fue procesada con el programa anteriormente mencionado y se obtuvieron tablas de frecuencias absolutas y relativas para cada una de las preguntas 
y cada uno de los encuestados. Con estos datos se elaboraron cuadros y gráficos para cada una de las subdimensiones consideradas en el análisis.

Para la presentación de los resultados globales para cada una de las dimensiones y subdimensiones, se diseñó la Tabla 4, mostrada a continuación.

Tabla 4. Valoración cualitativa de la gestión por dimensión y subdimensión

\begin{tabular}{|c|c|c|c|c|c|}
\hline OPCIONES & $\begin{array}{c}\text { CATEGORÍAS DE } \\
\text { RESPUESTAS }\end{array}$ & $\begin{array}{l}\text { FRECUENCIA } \\
\text { ABSOLUTA }\end{array}$ & $\begin{array}{l}\text { FRECUENCIA } \\
\text { RELATIVA }\end{array}$ & $\begin{array}{c}\text { FRECUENCIA } \\
\text { RELATIVA } \\
\text { ACUMULADA }\end{array}$ & $\begin{array}{l}\text { VALORACIÓN } \\
\text { CONFORMIDAD }\end{array}$ \\
\hline 1 & $\begin{array}{l}\text { Totalmente en } \\
\text { desacuerdo }\end{array}$ & & & & \\
\hline 2 & En desacuerdo & & & & \\
\hline 3 & $\begin{array}{l}\text { Ni de acuerdo ni en } \\
\text { desacuerdo }\end{array}$ & & & & \\
\hline 4 & De acuerdo & & & & \\
\hline 5 & $\begin{array}{l}\text { Totalmente de } \\
\text { acuerdo }\end{array}$ & & & & \\
\hline TOTALES & & & & & \\
\hline
\end{tabular}

En la Tabla 4, la frecuencia absoluta representa el número de respuestas totales obtenido de la subdimensión diagnosticada en cada una de las categorías para cada dimensión. La frecuencia relativa es el porcentaje que representa el total de respuestas de cada categoría en relación con el total de respuestas de la subdimensión, y la frecuencia relativa acumulada constituye la sumatoria de las frecuencias relativas de cada categoría.

Para la emisión de un juicio uniforme en la valoración cualitativa de la subdimensión diagnosticada respecto a lo establecido en las normas se utilizó la siguiente clasificación, de acuerdo a la concentración de respuestas en las opciones 1, 2 y 3 o 4 y 5, tal como se presenta en la Tabla 5.

Tabla 5. Valoración cualitativa de conformidad de la subdimensión diagnosticada

\begin{tabular}{|c|c|c|}
\hline \multicolumn{2}{|c|}{ CONCENTRACIÓN DE RESPUESTAS } & \multirow{2}{*}{$\begin{array}{c}\text { Valoración cualitativa de conformidad de } \\
\text { la subdimensión diagnosticada respecto } \\
\text { a la norma }\end{array}$} \\
\hline $\begin{array}{c}\text { OPCIONES } 1,2 \text { y } 3 \\
(\%)\end{array}$ & $\begin{array}{c}\text { OPCIONES 4, } 5 \\
\text { (\%) }\end{array}$ & \\
\hline$[30$ a 100$]$ & [0 a 70) & INCONFORME \\
\hline [0 a 30) & [70 a 100] & CONFORME \\
\hline
\end{tabular}

Es decir, para una dimensión en particular, si la frecuencia relativa acumulada de respuestas concentradas en las opciones 1, 2 y 3 se encuentra entre [30 y 100], la valoración de la gestión actual en relación con lo establecido en la Norma para esa subdimensión es INCONFORME. Si está entre [0 y 30) se considera CONFORME. 


\section{Resultados y discusión}

\subsection{Conformidades y no conformidades}

A continuación se presenta la Tabla 6 para la valoración de las conformidades y no conformidades encontradas.

Tabla 6. Valoración de conformidad de la gestión por subdimensión, para las dimensiones Calidad, Ambiente y SSO

\begin{tabular}{|c|c|c|c|}
\hline \multirow{2}{*}{ SUBDIMENSIONES } & \multicolumn{3}{|c|}{$\begin{array}{l}\text { VALORACIÓN DE CONFORMIDAD DE LA GESTIÓN POR SUBDIMENSIÓN, } \\
\text { PARA LAS DIMENSIONES CALIDAD, AMBIENTE Y SSO }\end{array}$} \\
\hline & $\begin{array}{l}\text { ISO } 9001: 2015 \\
\text { CALIDAD (\%) }\end{array}$ & $\begin{array}{l}\text { ISO 14001: } 2015 \\
\text { AMBIENTE (\%) }\end{array}$ & $\begin{array}{l}\text { OSHAS 18001: } 2015 \\
\text { SSO (\%) }\end{array}$ \\
\hline $\begin{array}{l}\text { CONTEXTO DE LA } \\
\text { ORGANIZACIÓN }\end{array}$ & $\begin{array}{l}\text { CONFORME } \\
17,2\end{array}$ & $\begin{array}{l}\text { INCONFORME } \\
54,5\end{array}$ & $\begin{array}{l}\text { INCONFORME } \\
100\end{array}$ \\
\hline LIDERAZGO & $\begin{array}{l}\text { CONFORME } \\
23,8\end{array}$ & $\begin{array}{l}\text { INCONFORME } \\
33,9\end{array}$ & $\begin{array}{l}\text { INCONFORME } \\
42,8\end{array}$ \\
\hline PLANIFICACIÓN & $\begin{array}{l}\text { INCONFORME } \\
35,5\end{array}$ & $\begin{array}{c}\text { INCONFORME } \\
49,9\end{array}$ & $\begin{array}{c}\text { INCONFORME } \\
45,9\end{array}$ \\
\hline APOYO & $\begin{array}{c}\text { CONFORME } \\
29,4 \\
\end{array}$ & $\begin{array}{c}\text { INCONFORME } \\
36,3\end{array}$ & $\begin{array}{c}\text { INCONFORME } \\
34,0\end{array}$ \\
\hline OPERACIÓN & $\begin{array}{c}\text { CONFORME } \\
21,9 \\
\end{array}$ & $\begin{array}{c}\text { INCONFORME } \\
44,4\end{array}$ & $\begin{array}{c}\text { INCONFORME } \\
44,5\end{array}$ \\
\hline $\begin{array}{l}\text { EVALUACIÓN } \\
\text { DESEMPEÑO }\end{array}$ & $\begin{array}{c}\text { INCONFORME } \\
36,6\end{array}$ & $\begin{array}{c}\text { INCONFORME } \\
61,1\end{array}$ & $\begin{array}{c}\text { INCONFORME } \\
53,0\end{array}$ \\
\hline MEJORA & $\begin{array}{c}\text { CONFORME } \\
13,3\end{array}$ & $\begin{array}{c}\text { CONFORME } \\
13,3\end{array}$ & $\begin{array}{c}\text { CONFORME } \\
16,7\end{array}$ \\
\hline
\end{tabular}

Como se puede observar, las mayores conformidades de la gestión actual de los laboratorios públicos y privados dedicados a la producción de biofungicidas con lo establecido en las normas consideradas para las distintas subdimensiones se obtuvieron en la dimensión CALI$\mathrm{DAD}$, donde, de las siete subdimensiones evaluadas cinco resultaron conformes, es decir, que la concentración de respuestas en las opciones 1, 2 y 3 fue inferior a 30 \%. Estas fueron: contexto de la organización, liderazgo, apoyo, operación y mejora.

En las subdimensiones Planificación y Evaluación del desempeño la valoración de conformidad resultó ser inconforme, esto es, que la concentración de respuestas en las opciones 1, 2 y 3 fue de $35,5 \%$ y $36,6 \%$, respectivamente.

Respecto a las dimensiones AMBIENTE y SSO, solo en la subdimensión Mejora se encontró conformidad con una concentración de respuestas en las opciones 1, 2 y 3 de 13,3 \% y 16,7 \%, respectivamente; mientras que para las otras subdimensiones la valoración resultó ser inconforme, ya que la concentración de las respuestas en las opciones 1, 2 y 3 fue superior al 30 $\%$, encontrándose los mayores porcentajes de inconformidad en la subdimensión Evaluación del desempeño, lo cual permite inferir acerca de la necesidad de establecer procedimientos y mecanismos de retroalimentación que conlleven a los laboratorios a una permanente revisión y mejora de sus procesos. 
De igual manera, se presenta la Tabla 7 donde se podrán observar los resultados obtenidos por subdimensión para cada una de las dimensiones consideradas por laboratorio. La primera columna identificada como SD corresponde a las siete subdimensiones consideradas. Estas fueron: CO: Contexto de la organización; L: Liderazgo; P: Planificación; A: Apoyo; O: Operación; ED: Evaluación del desempeño y M: Mejora; y en la última fila, el promedio ponderado por laboratorio (PP/L). En la segunda columna identificada como D, se presentan las dimensiones: Calidad (C), Ambiente (A) y SSO.

Las columnas identificadas como A, B, C, D, E, F, G, H, I, corresponden a los laboratorios y la última columna (P), al promedio obtenido para cada subdimensión en cada una de las dimensiones consideradas.

Tabla 7. Valores promedios por subdimensión y por laboratorio, para las dimensiones Calidad, Ambiente y SSO

\begin{tabular}{|c|c|c|c|c|c|c|c|c|c|c|c|}
\hline \multirow{2}{*}{ SD } & \multirow{2}{*}{ D } & \multicolumn{9}{|c|}{ LABORATORIOS } & \multirow{2}{*}{$\mathrm{P}$} \\
\hline & & A & B & C & D & $E$ & $F$ & G & $\mathrm{H}$ & I & \\
\hline \multirow{3}{*}{$\mathrm{CO}$} & C & 4,5 & 4,3 & 4,5 & 3,5 & 3,9 & 4,7 & 4,4 & 3,9 & 3,8 & 4,2 \\
\hline & $A$ & 4,1 & 3,9 & 3,6 & 3,5 & 3,4 & 1,0 & 4,2 & 1,9 & 2,1 & 3,1 \\
\hline & SSO & 1,0 & 1,0 & 1,0 & 1,0 & 1,0 & 1,0 & 1,0 & 1,0 & 1,0 & 1,0 \\
\hline \multirow{3}{*}{$\mathrm{L}$} & $\mathrm{C}$ & 4,1 & 4,0 & 2,6 & 3,7 & 3,5 & 5,0 & 4,8 & 4,1 & 4,0 & 4,0 \\
\hline & $A$ & 4,1 & 3,4 & 2,8 & 3,7 & 3,5 & 4,9 & 4,4 & 4,0 & 4,1 & 3,9 \\
\hline & sSO & 3,7 & 3,3 & 3,9 & 3,4 & 3,3 & 4,9 & 4,0 & 3,8 & 3,7 & 3,8 \\
\hline \multirow{3}{*}{$\mathrm{P}$} & $\mathrm{C}$ & 3,8 & 4,1 & 3,0 & 3,1 & 3,3 & 4,2 & 4,2 & 3,8 & 3,9 & 3,7 \\
\hline & $A$ & 3,5 & 3,5 & 2,3 & 2,8 & 3,3 & 3,7 & 3,7 & 3,6 & 3,5 & 3,3 \\
\hline & SSO & 3,2 & 4,0 & 2,7 & 3,1 & 3,3 & 5,0 & 4,1 & 3,3 & 3,2 & 3,6 \\
\hline \multirow{3}{*}{ A } & $\mathrm{C}$ & 3,9 & 3,9 & 3,5 & 2,8 & 3,6 & 4,5 & 4,3 & 4,3 & 4,1 & 3,9 \\
\hline & $A$ & 3,7 & 3,9 & 3,4 & 2,7 & 3,6 & 4,5 & 4,1 & 3,8 & 3,7 & 3,7 \\
\hline & SSO & 3,4 & 4,0 & 3,2 & 2,7 & 3,7 & 4,6 & 4,0 & 4,2 & 4,1 & 3,8 \\
\hline \multirow{3}{*}{0} & $\mathrm{C}$ & 4,0 & 3,8 & 3,4 & 4,0 & 3,7 & 5,0 & 4,5 & 4,5 & 4,5 & 4,5 \\
\hline & $A$ & 2,8 & 3,8 & 2,8 & 3,6 & 3,8 & 4,3 & 4,1 & 3,1 & 3,0 & 3,1 \\
\hline & SSO & 2,4 & 3,6 & 2,6 & 3,6 & 3,1 & 3,8 & 3,2 & 3,7 & 3,4 & 3,3 \\
\hline \multirow{3}{*}{ ED } & $\mathrm{C}$ & 4,0 & 3,7 & 2,8 & 3,3 & 3,5 & 4,6 & 4,8 & 3,8 & 3,7 & 3,8 \\
\hline & A & 3,2 & 3,3 & 2,8 & 3,1 & 3,5 & 4,3 & 4,6 & 2,2 & 2,3 & 3,2 \\
\hline & SSO & 3,1 & 3,5 & 3,0 & 3,1 & 3,5 & 4,6 & 4,5 & 3,9 & 3,8 & 3,7 \\
\hline \multirow{3}{*}{$M$} & $\mathrm{C}$ & 4,2 & 4,0 & 2,0 & 4,2 & 4,0 & 5,0 & 4,2 & 5,0 & 4,8 & 4,2 \\
\hline & $A$ & 4,2 & 4,0 & 2,0 & 4,2 & 4,0 & 5,0 & 4,2 & 5,0 & 4,8 & 4,2 \\
\hline & SSO & 4,0 & 4,0 & 2,0 & 4,0 & 4,0 & 5,0 & 4,0 & 5,0 & 4,8 & 4,1 \\
\hline \multirow{3}{*}{ PP/L } & $\mathrm{C}$ & 4,0 & 3,9 & 3,2 & 3,6 & 3,6 & 4,8 & 4,5 & 4,2 & 4,2 & \\
\hline & $\mathrm{A}$ & 3,5 & 3,6 & 2,9 & 3,2 & 3,5 & 4,1 & 4,2 & 3,2 & 3,2 & \\
\hline & SSO & 3,1 & 3,6 & 2,9 & 3,0 & 3,4 & 4,5 & 3,9 & 3,7 & 3,6 & \\
\hline
\end{tabular}

En la Tabla 7 se evidencia que en todas las subdimensiones consideradas los mayores valores promedio se obtuvieron en la dimensión CALIDAD, lo cual permite inferir que existe un gran interés por parte de la dirección de los laboratorios en los aspectos relativos a la gestión de la calidad y que existe la necesidad de tomar acciones para el mejoramiento de la gestión ambiental y de seguridad y salud ocupacional. 
De acuerdo con los resultados obtenidos se puede afirmar que los laboratorios considerados en el estudio poseen una gran fortaleza para la adopción de la norma ISO 9001:2015, en virtud de que se cumplen, de acuerdo con la opinión de los expertos, con muchos de los requisitos de la misma.

Por otro lado, se evidencia la necesidad de prestar especial atención a las dimensiones AMBIENTE Y SSO por cuanto constituyen pilares fundamentales para la sostenibilidad de la gestión en la producción de biofungicidas.

Siendo el interés primario del uso de biofungicidas contribuir con la disminución de la contaminación del ambiente y contribuir con la seguridad agroalimentaria, su producción debe responder a una gestión ambiental y de seguridad y salud ocupacional acorde con sus principios fundamentales.

\subsection{Diagnóstico situacional}

A continuación se presentan las fortalezas, debilidades, oportunidades y amenazas para que los laboratorios adopten una propuesta de gestión fundamentada en las normas consideradas en el presente estudio.

\section{Fortalezas}

- Tienen conocimiento y comprenden los aspectos externos e internos que son pertinentes para el propósito y dirección estratégica de la organización y que afectan a su capacidad para lograr los resultados previstos en cuanto a la calidad.

- Cuentan con el liderazgo y compromiso de la gerencia para promover en su dirección estratégica la adopción de medidas que coadyuven al mejoramiento de la gestión de los laboratorios en Calidad, Ambiente y SSO.

- Tienen establecido un control operacional para la producción de biofungicidas conforme con muchos de los lineamientos de la norma ISO 9001:2015.

- Cuentan con personal gerencial y técnico altamente calificado y especialistas en el área de producción de biofungicidas.

- Tienen la disposición y creen en la necesidad de la evaluación de su desempeño como una herramienta para el mejoramiento continuo de sus operaciones.

- Cuentan con un personal directivo comprometido con el mejoramiento continuo de sus operaciones.

- Cuentan con la infraestructura y dotación de equipos requeridos para la producción artesanal de biofungicidas.

- Aplican los principios de las Buenas Prácticas de Fabricación (BPF) y Buenas Prácticas de Laboratorio (BPL) para garantizar la calidad e inocuidad de los productos elaborados.

\section{Debilidades}

- Poseen poco conocimiento de las normas consideradas y del contexto externo e interno en lo concerniente a la gestión ambiental y de seguridad y salud ocupacional.

- Carecen de una cultura organizacional alineada con los principios sobre los que se fundamentan las normas consideradas.

- No disponen de una política integrada de Calidad, Ambiente y de SSO. 
- No cuentan con un procedimiento para la planificación de los riesgos y oportunidades.

- No cuentan con un procedimiento para llevar a cabo las actividades de: identificación de los aspectos ambientales y evaluación de los impactos ambientales asociados y para la identificación, acceso y entendimiento de los requisitos legales.

- Carecen de procedimientos estandarizados para la elaboración y mantenimiento de la información documentada que sea pertinente en Calidad, Ambiente y SSO.

- No tienen definidos criterios de evaluación, selección, seguimiento del desempeño y evaluación de proveedores externos; así como acciones de respuesta planificadas ante posibles emergencias y evaluación y revisión periódica de los procesos y de dichas acciones en calidad, ambiente y SSO.

- No disponen de un proceso sistemático de revisión y mejoramiento continuo de su gestión.

- Carecen de mecanismos de retroalimentación acerca de la satisfacción de sus clientes y de auditoría interna de sus organizaciones.

\section{Oportunidades}

- La necesidad de garantizar la seguridad agroalimentaria y la salud de las personas.

- La incidencia del uso de biofungicidas en la disminución del uso de agroquímicos.

- El crecimiento del mercado de biocontroladores.

- El entorno regulatorio del sector de producción de biofungicidas.

- La carencia de reglas claras, adecuadas y transparentes para el registro de los biofungicidas.

- La carencia de protocolos oficiales estandarizados para la producción de biofungicidas y su control.

- El comportamiento preferencial de los productores agrícolas hacia el uso de los agroquímicos.

- Inestabilidad económica del país lo cual incide en los costos de adquisición, reparación y reposición de equipos y repuestos, por lo que se requiere mantener un control estricto de las operaciones para garantizar el uso y mantenimiento adecuado de los mismos.

\section{Amenazas}

- Inexistencia de una política de Estado que incentive la producción, registro y comercialización de biofungicidas.

\subsection{Propuesta}

La gestión sostenible para la producción de biofungicidas está referida al compromiso con los principios del desarrollo sostenible, satisfacción de las necesidades y expectativas de los clientes y partes interesadas, desarrollo e implementación de sistemas basados en las buenas prácticas y sistemas de gestión de calidad, medioambiente, prevención, seguridad; así como con cualquier otro que coadyuve al mejoramiento continuo y aseguramiento de la calidad de los procesos y productos.

Bajo esa premisa se elaboró la propuesta esquematizada en la Figura 1, la cual consiste en la adopción de un enfoque sistémico de gestión para la producción de biofungicidas, concibiéndola como un proceso de administración y dirección de su funcionamiento y desarrollo, que debe adecuar su quehacer y ser con el deber ser. 
Figura 1. Gestión sostenible para la producción de biofungicidas.

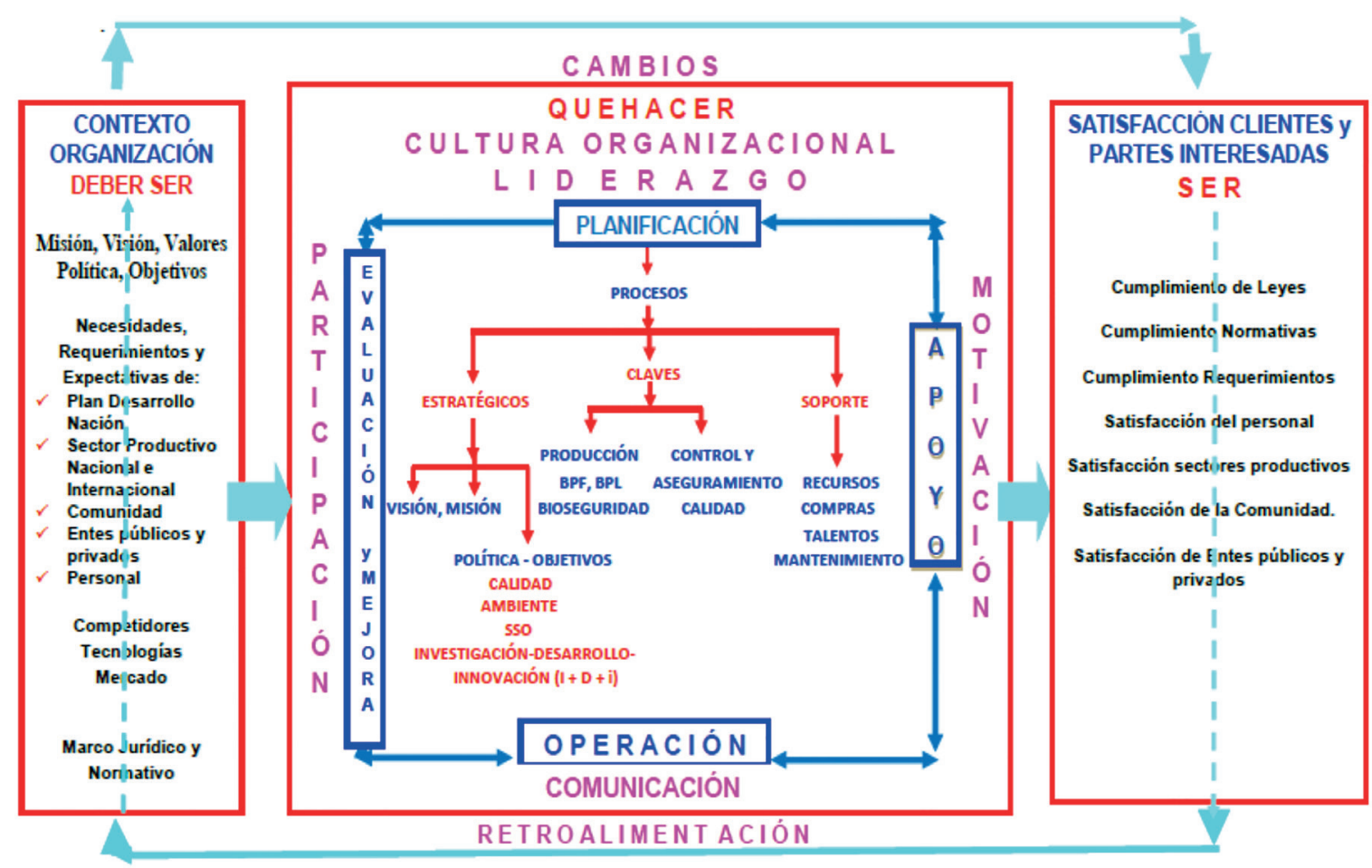

El Deber ser constituye el contexto de la organización y se define en función de los factores que influyen en el propósito, objetivos y sostenibilidad de la organización. Considera factores internos tales como los valores, cultura, conocimiento y desempeño de la organización y factores externos como entornos legales, tecnológicos, de competitividad, de mercados, culturales, sociales y económicos; así como las necesidades, requerimientos y expectativas de los clientes y partes interesadas.

El Ser está representado por los resultados del Quehacer de la organización: nivel de satisfacción de las necesidades y expectativas del entorno interno y externo, nivel de cumplimiento de las leyes, normativas y requerimientos establecidos en el Deber ser.

El Quehacer es el conjunto de procesos que deben desarrollarse para el cumplimiento del Deber Ser, aplicando de manera continua e interactiva el ciclo Planificar, Hacer, Verificar y Actuar, de acuerdo con los requisitos establecidos en las normas ISO 9001:2015, 14001:2015 y OSHAS 18001:2007,tomadas como referencia.

El enfoque propuesto conlleva a: 1) la vinculación permanente con el contexto para diagnosticar, pronosticar y establecer sus necesidades, requerimientos y expectativas; así como para evaluar su nivel de satisfacción y cumplimiento; 2) Un proceso de creación y mantenimiento de una cultura organizacional basada en el liderazgo, la participación, la motivación y la comunicación, con el propósito de promover un clima organizacional de solidaridad y trabajo en equipo; para garantizar el desarrollo del sentido de pertenencia y compromiso con la misión, visión, política y objetivos de la organización, por parte del personal; 3) Un proceso sistematizado de planificación, apoyo, operación, evaluación y mejora, en el Quehacer de los laboratorios y empresas, para garantizar la realización de los procesos estratégicos, claves u operativos y 
de soporte, conducentes al cumplimiento de su misión, la cual es producir biofungicidas con criterio de sostenibilidad.

De acuerdo con esta propuesta, la sostenibilidad de la gestión constituye uno de los procesos estratégicos que debe ser asumido y definido por la Alta Dirección de los laboratorios y empresas que producen biofungicidas, es decir, la gestión integrada de la calidad, del ambiente y de la seguridad y salud ocupacional, bajo los lineamientos de las normas consideradas en el estudio es una decisión estratégica de la organización y debe formar parte de su política.

La materialización de este enfoque de gestión requiere de un proceso gerencial con una perspectiva de futuro que responda a las necesidades y exigencias de su entorno y donde se diseñen las estrategias y las estructuras de funcionamiento interno que hagan posible una eficaz y eficiente gestión de los procesos, recursos y talentos disponibles, bajo un liderazgo que propicie un ambiente de alta participación, comunicación, motivación y compromiso con una cultura de seguimiento y evaluación permanente de su funcionamiento para el logro de los objetivos establecidos por la organización.

La adopción de la propuesta por parte de los laboratorios requiere la implementación de algunas estrategias, entre las cuales están:

a. Integración de los sistemas de gestión de la Calidad, Ambiente, Seguridad y Salud Ocupacional e Innovación, de acuerdo con los lineamientos de la Norma UNE 66177:2005 (AENOR, 2005).

b. Adopción de la Norma UNE 166002:2014, relativa a la gestión de la Investigación, Desarrollo e Innovación, como estrategia para sistematizar las actividades de I+D+i (AENOR, 2014).

c. Estandarización en la aplicación de las Buenas Prácticas de Fabricación (BPF), Buenas Prácticas de Laboratorios (BPL) y medidas de bioseguridad para la producción de biofungicidas.

d. Definición e implantación de una política integrada -Calidad, Ambiente, SsO- de la organización y aseguramiento del compromiso de la gerencia.

e. Formulación de un plan para satisfacer la política definida.

f. Normalización de los procesos a través del desarrollo de procedimientos y registros.

\section{Conclusiones}

Se establecieron las conformidades y no conformidades de la gestión actual de los laboratorios para la producción de biofungicidas, encontrando conformidad en cinco de las siete subdimensiones evaluadas en la dimensión Calidad. Para las dimensiones Ambiente y SSO, seis de las siete subdimensiones evaluadas resultaron inconformes.

Se determinaron las fortalezas, debilidades, oportunidades y amenazas para que los laboratorios adopten la propuesta de gestión diseñada, en función de las conformidades e inconformidades encontradas en el diagnóstico y en los beneficios que se derivan de su adopción.

Se generó una propuesta de gestión fundamentada en los lineamientos metodológicos de las normas ISO 9001:2015, ISO 14001:2015 y OSHAS 18001:2007, para la gestión sostenible de la producción de biofungicidas y fortalecimiento del sector de bioinsumos agrícolas del país, la cual consiste en la adopción por parte de los laboratorios de un enfoque sistémico de administración, dirección y desarrollo, que adecúa su Quehacer y Ser con el Deber ser. 


\section{Recomendaciones}

- Promover a nivel de todo el sector de bioinsumos agrícolas del país la adopción de una filosofía de gestión basada en el mejoramiento continuo, la cual no es más que un enfoque de administración y gerencia que interpreta a las organizaciones como sistemas abiertos dirigidos a satisfacer las necesidades y expectativas de sus clientes internos y externos, a través del control de procesos, eliminación del desperdicio, optimización de recursos, uso de herramienta estadísticas para el seguimiento y evaluación de los procesos y, quizás lo más importante, el compromiso de quienes lideran la organización para asumir el mejoramiento continuo y la autoevaluación como parte esencial de la cultura organizacional.

- Sensibilizar a todo el personal de los laboratorios, sobre la necesidad de adoptar la Gestión de la Calidad, Ambiente y SSO, como una estrategia para el cambio de la organización y cumplimiento de su misión y visión.

- Sensibilizar al personal directivo de los laboratorios sobre la necesidad de asumir la formación y capacitación gerencial como una estrategia para el cambio de la organización y búsqueda de la excelencia administrativa, en términos de la pertinencia y de la calidad de la gestión.

- Desarrollar herramientas para la organización, contabilización y análisis de los costos y beneficios que se generan de la implementación de la propuesta.

- Promover y contribuir con la formulación de políticas, normas y reglamentos que regulen la producción, registro y comercialización de agentes de control biológico y contemplen el financiamiento y protección de la producción nacional como aspectos fundamentales para el crecimiento y desarrollo sostenible del sector de bioinsumos agrícolas del país.

\section{Referencias}

Arcia, M. y Bautista, L. (2009). Control biológico en cultivo con hongos antagonistas y entomopatógenos. Caracas, Venezuela: CITECI.

Arias, F. (2006). El proyecto de Investigación, Introducción a la metodología científica. (5a ed). Caracas, Venezuela: Episteme.

Asociación Española de Normalización y Certificación. AENOR. (2005). Norma Española UNE 66177. Sistemas de gestión. Guía para la integración de los sistemas de gestión.

Asociación Española de Normalización y Certificación. AENOR. (2014). Norma Española UNE 166002. Gestión de la I+D+i: Requisitos del Sistema de Gestión de la I+D+i. [Documento en Línea]. Consultado el 27 de agosto de 2017 en https://es.scribd.com/doc/268148089/UNE-166002-2014-ESP

Balestrini, M. (2001). Cómo se Elabora el Proyecto de Investigación. (5a. ed.). Caracas, Venezuela: BL Consultores Asociados.

Cock, M. (1996). Control biológico de malezas. En: Manejo de Malezas para Países en Desarrollo. (Estudio FAO Producción y Protección Vegetal - 120). Organización de las Naciones Unidas para la Agricultura y la Alimentación. (Ed). Roma. [Libro en línea]. Recuperado el 05 abril de 2017 en: http:// www.fao.org/docrep/T1147S/t1147s0d.htm\#capítulo 9.control biológico de malezas

Chandler, D.; Bailey, A.; Tatchell, G.; Davidson, G.; Greaves, J., y Grant, W. (2011). The development, regulation and use of biopesticides for integrated pest management. Philosophical Transactions of the Royal Society B: Biological Sciences, 366(1573), 1987-1998. [Documento en línea]. Recuperado el 20 de marzo de 2017 en: https://www.ncbi.nlm.nih.gov/pubmed/21624919 
Falconí, C. (2014). Control biológico de enfermedades de plantas en Ecuador. En Bettiol, W., Rivera, M.; Mondino, P.; Montealegre, J. y Colmenárez, Y. (Eds.). Control biológico de enfermedades de plantas en América Latina y el Caribe. Facultad de agronomía. Universidad de la República. Montevideo, Uruguay. [Libro en línea]. Recuperado el 17 de marzo de 2017 en: https://www. researchgate.net/publication/272086409_Control_Biologico_de_Enfermedades_de_Plantas_en_ America_Latina_y_el_Caribe

Hernández, R., Fernández, C. y Baptista, P. (2014). Metodología de la Investigación. (6ª ed.). CDMX, México: McGraw-Hill.

Infostat. (2002). Infostat versión 1.1. Grupo Infostat, FCA. Argentina: Universidad Nacional de Córdoba.

Instituto Nacional de Salud Agrícola Integral. INSAI. (2016). Requisitos para el registro de biocontroladores. [Documento en línea]. Recuperado el 17 de marzo de 2017 en http://www.insai.gob.ve/wpcontent/uploads/2016/08/F-1-REQUISITOS-PARA-EL-REGISTRO-DE-BIOCONTROLADORES.pdf

Jaronski, S. (2013). Mass Production of Entomopathogenic Fungi: State of the Art. [Documento en línea]. Recuperado el 26 de junio de 2017 en https://www.researchgate.net/publication/281766568_ Mass_Production_of_Entomopathogenic_Fungi_State_of_the_Art

Organización de las Naciones Unidas para la Agricultura y la Alimentación. (FAO). 2006. "Inocuidad de los alimentos y sanidad animal y vegetal: Tendencias y desafíos para América Latina y el caribe". En: 29a Conferencia Regional de la FAO para América Latina y El Caribe. Caracas, Venezuela, 2428 de abril de 2006. [Documento en Línea]. Recuperado el 21 de mayo de 2017 en: http://www. fao.org/docrep/meeting/021/j7447s.pdf

Occupational Health and Safety Assessment Series. OSHAS. (2008). Norma Internacional OSHAS 18001:2008. Sistema de Gestión en Seguridad Industrial y Salud Ocupacional.

Organización Internacional para la Normalización (ISO). (2015a). Norma Internacional ISO 9001:2015. Sistemas de gestión de la calidad. Requisitos.

Organización Internacional para la Normalización (ISO). (2015b). Norma Internacional ISO 14001:2015. Sistemas de gestión ambiental. Requisitos con orientación para su uso.

Paleólogos, M. y Flores, C. (2014). Principios para el manejo ecológico de plagas. En Sarandón, S. y Flores, C. (Ed). Agroecología: bases teóricas para el diseño y manejo de Agroecosistemas sustentables. Facultad de Ciencias Agrarias y Forestales. Universidad Nacional de La Plata. Argentina. [Libro en línea]. Consultado el 12 de marzo de 2017 en http://www.mec.gub.uy/innovaportal/ file/75868/1/agroecologia.pdf

Reyes, A.; Rincón, G.; López, L.; Evangelista, Z. y Quiñones, E. (2015). Lucha entre microbios: una herramienta para el control de enfermedades de plantas. Revista Digital Universitaria. [Revista en línea]. 16 (11). Consultado el 27 de marzo de 2018 en http://www.revista.unam.mx/vol.16/ num11/art92/

Ruiz, C. (s. f). Confiabilidad. Programa Interinstitucional Doctorado en Educación. [Documento en Línea]. Consultado el 17 de enero de 2018 en http://www.carlosruizbolivar.com/articulos/archivos/ Curso\%20CII\%20\%20UCLA\%20Art.\%20Confiabilidad.pdf

Serrano, L. y Galindo, E. (2007). Control biológico de organismos fitopatógenos: un reto multidisciplinario. Revista Ciencia: enero-marzo 2007. [Revista en línea]. Consultado el 04 de abril de 2017 en http://www.ibt.unam.mx/Geg/lineas/Control\%20Biologico\%20Ciencia.pdf

Zambrano, C.; Goyo, Y.; Jiménez, M. y Zambrano, K. (2014). Control biológico de enfermedades de plantas en Venezuela. En Bettiol, W., Rivera, M., Mondino, P., Montealegre, J. \& Colmenárez, Y. (Eds.). Control biológico de enfermedades de plantas en América Latina y el Caribe. Facultad de agronomía. Universidad de la República. Montevideo, Uruguay. [Libro en línea]. Consultado el 17 de marzo de 2018 en https://www.researchgate.net/publication/272086409_Control_Biologico_ de_Enfermedades_de_Plantas_en_America_Latina_y_el_Caribe 